\title{
UN HOMENAJE A NUESTRO HÉROE. LECTURA DE LEON POMPA (POR J.M. SEVILLA)
}

\author{
Leon Pompa - José M. Sevilla
}

\begin{abstract}
Resumen: De la mano de L. Pompa, J.M. Sevilla traza un itinerario de estudios y contribuciones sobre Vico que el investigador escocés ha delineado desde 1971 hasta su traducción al inglés y edición en 2002 de la Ciencia nueva primera. Se ofrece así, con voz prestada, un importante e indiscutible relato biobibliográfico de un estudioso e intérprete de Vico que ha engrandecido los estudios viquianos en lengua inglesa.

Palabras Clave: Vico, $350^{\circ}$ Aniversario, ciencia, epistemología, metafísica, historia, Ciencia nueva primera, L. Pompa.
\end{abstract}

\section{A tribute to our hero}

ABSTRACT: By the hand of L. Pompa, J.M. Seville draws up an itinerary of studies and contributions on Vico that the Scottish researcher has described since 1971 until his 2002 translation into English and edition of the first New science. It offers thus, with borrowed voice, an important and indisputable biobibliographical story of a scholar and interpreter of Vico that has enlarged Vichian studies in English language.

KEYWORDS: Vico, 350 th Anniversary, science, epistemology, metaphysics, history, First New science, L. Pompa.

\section{Un omaggio al nostro eroe}

RIASSunTo: Ripercorrendo i lavori di Leon Pompa, J.M. Sevilla traccia un itinerario di studi e contributi su Vico che il ricercatore scozzese ha realizzato dal 1971 fino alla sua traduzione in inglese della prima Scienza nuova nell'edizione del 2002. Viene in talo modo offerta, con voce per così dire presa a prestito, un'importante e indiscutibile storia biobibliografica di uno studioso e interprete di Vico che ha ampliato gli studi vichiani in lingua inglese.

PAROLE CHIAVE: Vico, $350^{\circ}$ anniversario, scienza, epistemologia, metafisica, storia, prima Scienza nuova, L. Pompa.

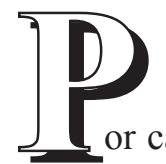

r causas mayores y ajenas a su voluntad, mi estimado profesor y amigo Leon Pompa no puede escribir un texto biobibliográfico como sería de desear y todos esperamos. No obstante, el profesor Pompa me ha manifestado su deseo de que un problema de salud no le impida estar presente en este «homenaje a nuestro héroe», de tal modo que me ha sugerido el que haga yo «una valoración» de su «contribución a los estudios sobre Vico». Para mí resulta un honor tal encargo. Solo

Este artículo responde a una invitación expresa por parte de la Dirección de la Revista para este volumen especial por el $350^{\circ}$ Aniversario del nacimiento de G. Vico, habiendo superado los criterios de valoración y del proceso de aceptación. 
espero estar a la altura de la tarea y ofrecer en esta Nota una digna idea acerca de su «principal contribución teorética y sobre las críticas de que haya sido objeto». ${ }^{1}$ Para ello no reseñaré ni evaluaré la contribución de un maestro, sino que enfocaré su contribución desde el ángulo particular y concreto de mi propia perspectiva -como, por otro lado, no podría ser de otro modo- de su lectura viquiana y de cómo muchas aportaciones de Pompa han influido en los estudios sobre Vico.

Conozco al prof. Pompa personalmente desde 1994, fecha en la que yo era un joven profesor y estudioso de Vico que, en aquel magnífico Convegno viquiano organizado por el Istituto Suor Orsola Benincasa, regido por Francesco De Sanctis, y celebrado en Nápoles del 1 al 3 de un frío diciembre, tuve la ocasión de conocerlo en persona. ${ }^{2}$ Pompa era ya, por entonces, una de las reputadas figuras extranjeras $^{3}$ a quien, como a tantos otros autores presentes, yo casi veneraba como a una divinidad sapiencial perteneciente al olimpo de estudiosos de Vico. Allí estaban en el Suor Orsola figuras míticas para mí como Agrimi, Badaloni, Battistini, Botturi, Cacciatore, Cantelli, Crifò, Giuliani, Mathieu, Pons, Tagliacozzo, Vitiello; algunas hoy lamentablemente desaparecidas, y envuelto en un largo elenco de reputados investigadores e intérpretes de Vico, entre los cuales nos movíamos algunos emergentes más jóvenes que yo, como Fabiani y Patella, y otros más o menos de mi edad, como Lilla. Entre los Maestros, resplandecía por afabilidad y simpatía en distancia corta Leon Pompa, por entonces profesor de Filosofía de la Universidad de Birmingham. ${ }^{4}$ Con uno y otros hablaba de filosofía, y sobre todo de Vico. Recuerdo algunas amenas conversaciones durante algún corto paseo o delante de un ristretto y un vasito de grappa tras la cena; y recuerdo cómo en cierto momento le comenté al prof. Pompa que me había sido de gran utilidad para mi Tesis Doctoral su ensayo de 1971 Vico's Theory of the Causes of Historical Change, publicado como $\mathrm{n}^{\mathrm{o}} 1$ de la serie de Monografias del Institute for Cultural Research. Rememoro que lo primero que me preguntó a continuación fue: «¿y qué le pareció?» «¿Cómo valora Usted aquel antiguo ensayo mío?» Quedé anonadado de que no solo no se me justificara su contenido, sino que además me solicitase, hace un

1. E-mail de L. Pompa a J.M. Sevilla, asunto: “Un abbraccio cordiale”, fecha 05/08/2018, hora: 18:51.

2. Las Actas del Congreso fueron publicadas por el ISOB en un imponente volumen a cargo de MARIO AGRIMI: Giambattista Vico nel suo tempo e nel nostro, CUEN, Nápoles, 1999; dedicado a la memoria de tres participantes que fallecieron en el ínterin de la publicación: A. Giuliani, E. Pii y G. Tagliacozzo.

3. En el citado volumen de Actas al cuidado de Agrimi se recoge en pp. 219-248 la contribución de Pompa titulada «Vico: imagination, naturalism, religion and reason». En este estudio, Pompa rastrea algunas líneas de investigación sobre Vico para plantear cómo puede ser resuelta la aparente contradicción entre ser aclamado este como un inaugurador de la Modernidad y también, a la vez, como la tardía culminación de la filosofía renacentista. «No me refiero, por supuesto, a que yo pueda resolver todos los problemas de interpretación asociados a un pensador tan complejo como Vico» -esgrime Pompa-.. «Mas espero poder establecer suficientes puntos como para proporcionar la base para una reevaluación crítica de, al menos, algunas líneas de interpretación». (Op. cit., p. 220).

4. L. Pompa, nacido en Edimburgo en 1933, fue desde 1961 profesor de Filosofía en la Universidad de la capital escocesa; y luego más tarde ha sido profesor en la Universidad de Birmingham, donde ha finalizado su carrera académica como Emeritus. 
cuarto de siglo, pero al igual que hoy, una evaluación de su contribución. A los continentales, y más aún a los meridionales, nos asombra esta capacidad anglosajona de sometimiento a la crítica, de permanente examen y evaluación. No lo recuerdo ya bien, pero debí de responderle que había llamado mi atención su exposición analítica de las causas y de las motivaciones que, según interpretaba de la doctrina de Vico, impulsan a los hombres a la evolución social. ¿Cómo pueden los hombres haber convenido en sociedad a partir de un estado bestial y desde hombres solitarios? Pues no solo de entablar relación social se trata, sino de estructurarla legalmente. Para llevar a cabo su evolución histórica «los hombres requieren no solamente de un contexto social, sino de un contexto social legalmente estructurado». ${ }^{5}$ Un interesante tema de charla compartido por entonces con la sabiduría filosófico-jurídica del hoy ausente Alessandro Giuliani.

La separación de Vico de los iusnaturalistas y de los contractualistas, a la hora de explicar los fundamentos de ese paso y de la forma del proceso, radicaba en que el napolitano no afirmaba la existencia de unas leyes racionales naturales a los hombres y entendidas por estos, ni la existencia de un pacto inicial en el estado de naturaleza. Pompa muestra, ya en ese primigenio ensayo, la posición de Vico al respecto del problema sistematizando las críticas que este lleva a cabo de las doctrinas iusnaturalistas: 1) la sociedad no puede surgir de un pacto inicial porque los "pactos" o acuerdos con valor jurídico presuponen un anterior ejercicio y comportamiento ya social; 2) el hombre no vive en una sociedad estructurada inicialmente por la razón humana: el derecho natural de las gentes no es el derecho natural de los filósofos, acontecido miles de años después de la fundación de las naciones. ${ }^{6} \mathrm{El}$ origen no es la convención, pero tampoco lo es la utilidad (Hobbes, Spinoza, Bayle). Frente a los utilitaristas, para Vico la utilidad es ocasión para el impulso social, pero no causa, es decir, no es su razón, causa o motivo ("cagione"). La confusión de lo histórico con lo filosófico invalidaba el juicio de teóricos del derecho natural (Grocio, Selden, Pufendorf), contractualistas y utilitaristas; pues, como los primeros, ofrecían una estática explicación estrictamente racionalista fundada sobre una concepción previamente idealista de la aequitas naturalis mediante la que abrazaban tanto la concepción de la naturaleza del hombre como el concepto mismo del contenido (histórico, positivo) del derecho. Este vicio de la razón sobre la historia les permitía afirmar, con boria dei dotti, un modelo de naturaleza humana estática, inmutable y eterna. Vico iba en contra de esa fundación histórica del derecho basa-

5. L. Pompa, Vico's Theory of the Causes of Historical Change (1971), Institute for Cultural Research (ICR Monograph Series $\mathrm{n}^{\circ}$ 1), Kent, 1979, p. 6. Cfr. de Pompa, por la misma fecha, «Vico's Science», History and Theory, X, 1, 1971, pp. 49-83 (traducido al italiano en el Bollettino del Centro di studi vichiani al año siguiente). Vid. J.M. SEVILla Fernández, Giambattista Vico: metafisicica de la mente e historicismo antropológico. (Un estudio sobre la concepción viquiana del hombre, de su mundo y de su ciencia), Publicaciones de la Universidad de Sevilla, Serie Filosofía y Letras no 110, Sevilla, 1988, pp. 398-399.

6. L. PomPA, op. cit., pp. 7-8. Vid. SN44 § 313. Cfr. J.M. SEVILLA, op. cit., pp. 399-400. 
do en una verdad racional necesaria, erigido sobre la "causa" de una naturaleza racional. Como dijera Isaiah Berlin, Vico siempre fue tremendamente concreto donde los iusnaturalistas eran pretenciosamente abstractos. ${ }^{7}$

Pompa me proporcionó entonces una de las principales claves para interpretar la idea del derecho como construcción-expresión histórica de la comunidad humana, naciente con ella: el derecho expresa la construcción "humana" de la sociedad, el proceso de conquista de una humanidad, es decir, de aquello que hace humano al hombre, a partir del salvajismo bestial y del estado de errar de fieras. Parafraseando a Pompa he dicho al respecto:

«La misma realidad jurídica, como manifestación de la compleja y dinámica (cambiante) naturaleza humana, expresión de la naturaleza histórica humana, excluye tanto una consideración intelectualista y apriorística del derecho cuanto de la naturaleza humana abstractamente considerada, creencia que indujera al error propio de la "vanidad de los doctos". Los teóricos del derecho natural solo supieron ver el contenido del derecho de una época concreta (cual era la suya propia), racional, civilizada, equitativa e iluminada, pero extendieron ahistóricamente esta concepción de forma universal para todas las épocas históricas de la humanidad, como si todo hubiera sido siempre igual». ${ }^{8}$

Esa visión iusnaturalista de una errada filosofía racionalista de la historia, precisamente, fue la que empujó a Vico hacia un problemático pero heroico y fructífero historicismo. Pompa explica que Vico considera la naturaleza humana cambiante, la entiende como un proceso de historicidad vinculado indeleblemente a una sucesión de fases de desarrollo que lo son de la mente humana y así, por tanto, de maneras de la mente en su desarrollo (por eso la mente humana tutta spiegata puede llevar a cabo la nueva indagación científica histórica de los principios acerca de la naturaleza -cambiante en realidad pero "común" en el disegno o "patrón" mentalde las naciones). Del mismo modo describe la guisa o la manera de desarrollo que siguen las sociedades, en las que el hombre no puede ser concebido distintamente de este proceso de desarrollo: de manera que su propia acción, tanto pensada como inconscientemente, condiciona las relaciones sociales, las cuales a su vez, puesto que el hombre se halla inmerso en ellas, motivan o condicionan las nuevas acciones. Esta

7. Cfr. I. BerLIN, Vico ed Herder. Due studi sulla storia delle idee, trad. it. e introd. de A. VerRI, Armando Armando Ed., Roma, 1978, p. 113 (ed. original inglesa en The Hogarth Press, Londres - Nueva York, 1976). Hay trad. española de C. González del Tejo: Vico y Herder. Dos estudios en la historia de las ideas, Cátedra, Madrid, 2000.

8. J.M. SEVILLA, op. cit., p. 406.

9. L. Pompa, A Study of the New Science, Cambridge U.P., Cambridge-Nueva York-Melburne, 1975. La $2^{\mathrm{a}}$ ed. en 1990 contiene un capítulo añadido por el Autor -a partir de las críticas realizadas- dedicado a los principios por los que se rige el desarrollo del derecho. Vid. citaciones del texto en Giambattista Vico. Studio sulla Scienza Nuova, trad. it., Armando Armando Ed., Roma, 1977, con introd. a la ed. it. de V. Mathieu, pp. 79, 130-132. El cap. 16 reproduce parte del material del artículo de 1971 sobre «La ciencia de Vico». 
intepretación, extraída de su famosa monografía Vico. A Study of the New Science, ${ }^{9}$ me acompañó en el final del cap. II de la Parte II de mi libro antes citado en nota, encadenado este a la perspectiva pompeana de que «la sucesión de cambios» a través de la serie que desarrolla el derecho natural «está determinada por la sucesión a través de la cual se desarrolla la naturaleza humana»; ${ }^{10}$ capítulo que finalizaba siguiendo a Pompa así:

«Proceso histórico visto tanto en términos de mente humana como de sociedad, proceso histórico-socio-cultural o proceso de la vida humana. El hombre es un ser de naturaleza social porque es mente; la sociedad se realiza en la historia, realizándose la naturaleza humana, como la mente se desarrolla históricamente. Metafísicamente entendido, su ser mente es condición de su ser social, e históricamente comprendido, por su realización, su ser social es condición para el desarrollo y despliegue de la mente».11

Un problema que ha ocupado constantemente a Pompa en relación con Vico ha sido el del conocimiento histórico. Así, en una contribución al importante volumen tagliacozziano de Vico and Contemporary Thougth, titulada Human Nature and the Concept of a Human Science, fruto de una ponencia congresual, Pompa trata acerca de «los dos principales argumentos filosóficos que subyacen en la concepción de Vico de una ciencia humana»; a saber: a) «que nuestro conocimiento del mundo de los fenómenos humanos puede ser tan riguroso y tan científico como nuestro conocimiento del mundo de los fenómenos naturales»; b) «que, en tanto que ciencia humana, ha de recurrir al conocimiento antecedente [previo, anticipado: "antecedent knowledge"] de la experiencia de lo que es ser humano», lo que permite una mayor inteligibilidad en los resultados de la ciencia humana que en los de cualquier ciencia natural. Estos argumentos, lejos de ser incompatibles entre sí, significan y constituyen según Pompa «una de las contribuciones más importantes de Vico a la filosofía». ${ }^{12} \mathrm{Y}$ para precisar ese carácter de verdadera "ciencia" respecto de la Scienza nuova, Pompa se centra en la propuesta de cognoscibilidad de los principios «a través» o «dentro de las modificaciones de la mente humana», como dice el propio Vico en su famoso parágrafo 331 de la Scienza nuova de 1744. De ahí la importancia de aquello que nuestro estudioso escocés denomina un «conocimiento experiencial antecedente» o previo en una ciencia humana de lo que es ser

10. Ibíd., p. 67.

11. J.M. SEVILLA, op. cit., p. 423.

12. L. Pompa, «Human Nature and the Concept of a Human Science», en G. Tagliacozzo, M. Mooney, D.P. Verene (EDs.), Vico and Contemporary Thougth, en New School for Social Research, n. 43, vols. 2: ns. 3 y 4, 1976; n. 3, pp. 434-445; cit., p. 434. [Edición también en Humanities Press, A.H. Nueva York, 1979; y trad. esp. de M.A. Díaz-Canedo y S. Mastrangello, Vico y el pensamiento contemporáneo, FCE, México, 1987). 
humano; lo que en la doctrina epistemológica viquiana podría comprenderse en tres puntos: 1) «que su ciencia depende en cierto modo del hacer humano»; 2) que nuestro conocimiento fáctico envuelve el recurso a nuestro conocimiento de las modificaciones de la mente humana; 3) que alcanzamos la certeza del segundo momento por un proceso de autorreflexión. ${ }^{13} \mathrm{El}$ conocimiento previo o antecedente es también fáctico ("factual knowledge"), una especie de antecedente teorético ("vero") que para ser científico requiere de su consecuente empírico ("certo"), y estar abierto a las demostraciones filológicas además de a las filosóficas. ${ }^{14}$ Según la interpretación de Pompa, que ya he declarado haber asumido en anteriores iniciales momentos de mis investigaciones, ${ }^{15}$ la apelación a un "conocimiento antecedente" en una ciencia humana no es un llamamiento a las propiedades "puramente formales" de la naturaleza humana, sino más bien «una apelación al conocimiento del contenido, del ser genético o social, de la naturaleza humana». ${ }^{16}$ La concepción epistémica antropológico-histórica de Vico se basa, pues, en la conjunción de dos clases de modificaciones: la genética (modificaciones psicológicas de la mente) y la histórica (los tres principios sociales necesarios para que emerja la humanidad civil). Esa condición histórico-social de "humanidad" hace a la naturaleza humana algo, al menos en parte, "condicionada" por el devenir y las circunstancias; de modo que invalida cualquier concepción de una naturaleza "fija" e "inmutable". Por esa misma razón, Pompa aduce que para la epistemología viquiana uno de sus imprescindibles principios es el de que «el conocimiento debe ser conocimiento causal», lo que en clave viquiana extraída de la Ciencia nueva significaría que atendiendo a las «modificazioni della mente humana» puede tenerse un conocimiento causal, es decir, ciencia. ${ }^{17}$

La interpretación de Pompa ha tenido a veces algunos objetores, como ha sido el caso de Isaiah Berlin, quien criticó a su colega británico que su vehemencia por demostrar el carácter verdaderamente epistémico de la Ciencia de Vico no le haría demostrar, en cambio, cómo otros hombres de una edad posterior pueden tener conocimiento verdadero de las necesidades sociales y de los objetivos de una mentalidad primitiva; o sea: ¿cómo penetrar en dicha realidad pasada, cuando las leyes de desarrollo son demasiado generales como para reconstruir o recrear fenómenos sociales y culturales específicos y concretos? Pompa argumenta que por "modificazioni" Vico quiere decir "necesidades y utilidades" sociales, comunales y no indivi-

13. Ibíd., p. 437.

14. Cfr. ibíd. pp. 439-440.

15. Vid. J.M. SevilLa, op. cit., pp. 240-244, siguiendo (y explicando) en esas páginas las intepretaciones a veces enfrentadas de Pompa y de Berlin.

16. Cfr. L. PomPA, «Human Nature and the Concept... », cit., pp. 439-440.

17. Cfr. L. Pompa, G. Vico. Studio sulla Scienza Nuova, cit., p. 174. Vid. de Pompa «Vico's Science», History and Theory, cit., e ID., "Vico and the Presuppositions of Historical Knowledge», en AA.VV., G. Vico's Science of Humanity, a cargo DE G. TAGLIACOzzo y D.P. Verene (EDS.), The Johns Hopkins U.P., Baltimore, pp. 125-40 (especialmente el apdo. IV en pp. 130-131). 
duales; a lo que Berlin critica que Pompa llegue a considerar la mente como una especie de conciencia social general, gobernada por unas leyes sobre las cuales puede fundarse la nueva ciencia. ${ }^{18}$

Un Estudio sobre la Scienza nuova, tan importante como el de Pompa, del que dijo Max Harold Fisch que se necesitaría más de una década para «asimilar»el libro, por fuerza tiene que haber generado debates y críticas. El propio Fisch elaboró una ponencia congresual como "respuesta" al libro de Pompa. ${ }^{19}$ Una argumentación sugerida por el libro, mas no contra el mismo («No creo estar en desacuerdo con Pompa en ninguno de los aspectos que considero esenciales en su argumento», reconoce Fisch; quien tiene solo algunas «dudas incidentales y comentarios»). En torno al problema de una ley de explicación histórica, Fisch esgrime que Pompa, intencionadamente, habría contribuido el primero -al menos en nuestra época- a potenciar, frente a una "filosofía especulativa de la historia", una "filosofía analítica de la historia":

«Aunque Pompa no utiliza este lenguaje, el principal argumento de su libro puede parafrasearse resumiéndolo como sigue: lo que se ha considerado como la aportación de Vico a la filosofía especulativa de la historia, era realmente el ejemplo arquetípico del modelo de ley inclusiva de explicación histórica en la filosofía analítica de la historia». ${ }^{20}$

Hasta la llegada del libro de Pompa ninguna historia de la filosofía de la ciencia se habría atrevido a incluir a Vico entre los serios epistemólogos o, mejor aún, "científicos" o hacedores del conocimiento científico. Mas, hasta hoy, tampoco -salvo pequeñas excepciones- han prestado mucha atención los historiadores y filósofos de la ciencia al descubrimiento de Vico. No por culpa del napolitano, ni tampoco del libro de Pompa, sino por la boria a veces de esos mismos historiadores.

Pompa ha defendido que Vico «propone un conjunto de condiciones mínimas que debe satisfacer cualquier cosa que pueda llamarse inteligiblemente una ciencia humana». ${ }^{21}$ Eso resultaría indiscutible. Cualquier objeto de discusión habría de plantearse ya el debatir si la propia ciencia nueva viquiana cumple con ese conjunto de "condiciones mínimas", pues de lo contrario no podría ser considerada una "cien-

18. Cfr. I. Berlin, Vico ed Herder, cit., Note, III, 13 (p. 128). En el "Prefacio del autor" (julio de 1975) Berlin agradece «al Dr. Leon Pompa que se prestara a discutir conmigo sus teorías sobre Vico», en especial las concepciones viquianas de «la ciencia y del conocimiento», que reconoce haberle sido muy útiles, si bien el libro de Pompa Vico: A Study of the New Science no pudo utilizarlo, pues, «por desgracia», se publicó estando ya el suyo en imprenta. (Un crítico a propósito de $A$ Study of the New Science ha sido RANDALl E. AuXIER: «Imagination and Historical Knowledge in Vico: A Critique of Leon Pompa's Recent Work», Humanitas, 10, 1997, pp. 26-49).

19. Cfr. M.H. Fisch, «Qué tiene que decir Vico a los filósofos contemporáneos?», en Vico y el pensamiento contemporáneo, cit., pp. 15-24.

20. Ibíd., p. 20. Cfr. M.H. Fisch, «Comentario a la ponencia del Profesor Pompa», ibíd., pp. 58-59 (la cita anterior entre paréntesis corresponde a la p. 58).

21. L. PomPA, «Respuesta del autor» [al Comentario de Fisch], ibíd., pp. 60-62; p. 60. 
cia humana". Si se tiene en consideración la interrelación lógica, causal y empírica que propone Vico (principios epistemológicos generales del verum-factum; fareconoscere; y principios propios de la ciencia nueva, como las proposiciones de los "Elementos", p.e. dignidades VIII, XI-XII, XIV, XV... LV-LVIII, LXII... etc., y especialmente LXIV-LXVI; o en "De los Principios" los tres principios comunes a todos los pueblos y que se convierten en condiciones socio-históricas de origen de cualquier -tanto real como posible- humanidad: religiones, matrimonios, sepulturas), entonces se comprende que «Vico puede admitir que las conclusiones de una ciencia tal están sujetas a modificaciones, siempre y cuando los presupuestos que la fundan sean tales que nos permitan entender su sentido humano».22 Según Pompa, si

«algunos de estos supuestos son de naturaleza fáctica, los cambios no tendrán que abarcar cada posible hecho, puesto que estos supuestos factuales tienen un alto nivel de generalidad y aún dejan a la investigación empírica el lugar indispensable que le corresponde en el establecimiento de las conclusiones de la ciencia».23

La indagación de la naturaleza del conocimiento histórico centrada en Vico ha llevado a Pompa a confrontar la teoría viquiana con otras destacables, como la hegeliana, ${ }^{24}$ la marxiana o, por ejemplo, la dendrognoseológica tagliacozziana. Así, de la confrontación entre las teorías de Vico y Marx acerca de la naturaleza del mundo histórico ha salido, en Estructura ontológica e historiográfica en Vico y Marx, un «Vico en posición de ofrecer una concepción más factible» de dicha naturaleza que la concepción de Marx. ${ }^{25}$

Por otro lado, una perspectiva de la concepción filosófica viquianizada por Giorgio Tagliacozzo en su propuesta de un arbor scientiae la aborda Pompa en su contribución de 1999 The Diachronic and the Synchronic, recogida en un homenaje póstumo a Tagliacozzo a cargo del malogrado Franco Ratto, en donde el estudioso edimburgués plantea la coexistencia entre modificaciones de la mente como imaginación y razón; a la vez que entre -digamos nosotros- una vía semántica y una vía sintáctica de la historia. ${ }^{26}$ Pretende Pompa

22. Ibíd., p. 61.

23. Ibídem.

24. Principalmente L. Pompa, «Vico and Hegel», en G. Tagliacozzo (Ed.), Vico. Past and Present, Humanities Press, Atlantic Higlands, 1981, vol. 2.

25. Cfr. la publicación original inglesa en el volumen compilado por Giorgio TAGLIACozzo bajo el título Vico and Marx. Affinities and Contrasts, Humanities Press, Nueva Jersey, 1983; y en su ya referida traducción al español de Sadie Ordales de la Garza en Vico y Marx. Afinidades y contrastes, FCE, México, 1990, pp. 66-80. De 1982 es su ensayo «La fantasia in Vico», en AA.VV., Leggere Vico, Spirali ed., Milán.

26. L. POMPA, «The Diachronic and the Synchronic», en F. RatTo (ED.), All'ombra di Vico. Testimonianze e saggi vichiani in ricordo di Giorgio Tagliacozzo, Edizioni Sestante, Ripatransone (A.P.), s.d. (1999), pp. 317-325. También es interesante, publicado igualmente por Ratto, de L. POMPA: «Theism and Vico's Philosophy and History of Humanity», en F. RaTto (ED.), Il mondo di Vico/Vico nel mondo, Edizioni Guerra, Perugia, 2000, pp. 271-82. 
«sugerir que Tagliacozzo tenía razón al pensar que Vico mostraba cómo lo sincrónico y lo diacrónico podían vincularse en una sola ciencia, mediante la idea de que las creencias dominantes en un sistema holístico proporcionan las necesarias condiciones previas conceptuales para el desarrollo de nuevas creencias dominantes en un sistema holístico diferente».

Sin embargo, opina que el fundador de New Vico Studies «estaba equivocado al pensar que Vico creía que diferentes ideas dominantes, o diferentes cuerpos de conocimiento basados en estas, también podrían coexistir». ${ }^{27}$ Por esa razón le parece a Pompa «improbable que su [de Tagliacozzo] viquianizado Árbol del Conocimiento, en el que esta concepción claramente ahistórica está integrada tanto en el tronco como en las ramas, sea una concepción que Vico pudiera haber aceptado».28

En todas sus contribuciones Pompa manifiesta su tesis de que la idea nuclear de la ciencia nueva es que todas las potencialidades para llegar a ser humanos no podrían desarrollarse si no fuera dentro de un contexto institucional, es decir, en un ámbito histórico de -aunque rudas y simples-instituciones sociales y civiles.

«Fracasaría por el hecho de que gran parte de nuestra naturaleza humana común consiste en la estructuración de nuestras actividades por medio de conceptos y creencias internamente vinculadas a tales instituciones y que no se podrían adquirir fuera de ese contexto». ${ }^{29}$

Es, como decimos, una tesis presente en la mayoría de los ensayos pompeanos, incluyendo entre estos sus contribuciones en español en Cuadernos sobre Vico (a cuyo Comité Científico de la revista pertenece desde sus comienzos), a saber: 1) La función del legislador en Giambattista Vico, ${ }^{30}$ texto en el que ofrece un análisis de la teoría viquiana de las instituciones jurídicas y del desarrollo social para extraer una -no definida por Vico- teoría sobre la función del legislador; 2) Hermenéutica metafísica y metafísica hermenéutica, ${ }^{31}$ que analiza dos modos de entender en la teoría hermenéutica viquiana la relación entre filología y filosofía, la cual origina dos diversas concepciones de la conexión entre hermenéutica y metafísica: en una, propia de la Scienza nuova de 1725, prima la hermenéutica metafísica -es decir, la interpretación depende de una metafísica-; en otra, característica acusada en la edición de 1744, predomina la definición de una her-

27. Ibíd., p. 324.

28. Ibíd., p. 325.

29. Ibídem.

30. Cuadernos sobre Vico, 5/8, 1995-1996, pp. 139-153.

31. Cuadernos sobre Vico, 7/8, 1997, pp. 141-166. En ese mismo año publica Pompa en inglés «Vico and Metaphysical Hermeneutics», en A. O'Hear (ED.), Verstehen and Humane Understanding, Cambridge U.P., Cambridge, 1997, Royal Institute of Philosophy Supplement 41, pp. 29-46. 
menéutica filosófica, más viable hoy frente a la anterior, pero en cualquier caso manifestaciones de que Vico advirtió la necesidad de disponer de una teoría hermenéutica; 3 ) sus amplias reseñas críticas Vico: política, moral y ciencia (recesión de obras de M. Lilla, de D.W. Black, y de H.S. Stone), Dos lecturas de Vico (sobre obras de M. Vaglio y de G. Mazzotta, respectivamente) y Precepto délfico del autoconocimiento. ${ }^{32}$

Entre otras aportaciones de Pompa a los estudios viquianos, especialmente en New Vico Studies y en el Bollettino del Centro di studi vichiani, ${ }^{33}$ junto a su Estudio de la Scienza nuova (libro en el que Pompa presenta una interpretación de las principales teorías filosóficas de la obra capital de Vico, «que sirve como una introducción analítica a ese texto» ${ }^{34}$ ), destacaremos la traducción y edición inglesa de la Scienza nuova de 1725. Pompa ha reconocido que considera esta edición inglesa de The First New Science su «más importante» contribución a los estudios de Vico, obra que le costó «años completarla» y que aún hoy se vende como libro de bolsillo al mismo y constante ritmo anualmente. ${ }^{35}$ La edición original inglesa de Pompa contiene una "Introduction" que inexplicablemente -y sin ninguna advertencia previa- fue incluida en español en la desafortunada reedición de la traducción - de 1995 de Rocío de la Villa- de la Ciencia nueva ¡de 1744!36

$\mathrm{Su}$ "Introduction" a la Ciencia nueva de 1725 deja claro desde el comienzo tanto la visión de una teoría científica, que Pompa atribuye a Vico, extensible a todas las cosas humanas, como la razón por la que esta traducción de la primera edición está acogida en la serie editorial "Cambridge Texts in the History of Political Thought" a cargo de Raymond Geuss y Quentin Skinner.

«Una de las principales lecciones que en relación con la teoría política enseña La Primera Ciencia Nueva es que cualquier teoría de este tipo ha de ubicarse dentro de una ciencia la cual incorpore tanto una filoso-

32. Respectivamente en Cuadernos sobre Vico, 9/10, 1998, pp. 329-332; ibíd., 13/14, 2001-2002, pp. 415-418; e ibíd., 17/18, 2004-2005, pp. 455-456.

33. Así, p.e., en el Bollettino del Centro di studi vichiani: «The Imaginative Universal», BCSV, XXXV, 2005; y «Reflection on the Ideal Eternal History», BCSV, XLI/2, 2011; o en New Vico Studies: el obituario «Isaiah Berlin 1909-1997», NVS, 16, 1998, pp. 129-136.

34. Giambattista Vico. Studio sulla Scienza Nuova, cit., p. 19 ("Prefazione").

35. Vico, The First New Science, editado por Leon PomPA, Cambridge U.P., Cambridge, 2002, con una "Introduction" en pp. xix-xxxviii. Antes había traducido y editado en la misma editorial universitaria una selección de textos viquianos: Vico. Selected Writings, Cambridge U.P., Cambridge, 1982, que cuenta con varias ediciones. Este último manual contiene textos en inglés de El método de estudios de nuestro tiempo, la Sabiduría primitiva de los Italianos, y de la primera y tercera Ciencia nueva; con un "Preface" de Pompa en pp. xi-xiv.

36. G. Vico, Ciencia Nueva, traducción y notas a cargo de R. DE LA Villa, prólogo de J.M. Romay Beccaría, introd. de L. Pompa, Ed. Tecnos, Madrid, 2006. El texto de Pompa fue traducido al español por Santiago Díaz Sepúlveda. Cfr. J. Martínez Bisbal, «Una reedición desafortunada», Cuadernos sobre Vico, 25/26, 2011-2012, pp. 240-241 («Aún siendo una buena introducción a la Sn25, por ello mismo no parece que pueda servir como introducción a la $\operatorname{Sn} 44[\ldots] » ;$ p. 241). 
fía como una historia de toda la naturaleza humana y de la completa práctica humana».37

Por eso, en su Introducción, Pompa comienza por rastrear «algunos puntos clave que condujeron a Vico hacia esta conclusión», para continuar discutiendo «algunos de los asuntos que se plantean desde la concepción a la que él finalmente llegó».$^{38}$ Entre estos asuntos, destaca el interés de Vico por lo social, presente desde sus primeros «escritos teoréticos», comenzando por las Oraciones Inaugurales en las que se enfatiza, por el bien de la sociedad, la importancia de una correcta interrelación entre intelectualidad y pueblo. ${ }^{39}$ De hecho:

«Uno de los objetivos principales de La Primera Ciencia Nueva es descubrir las causas de la estabilidad social y política, para permitirnos identificar y enmendar las inestabilidades en caso de que surgieran».40

Tal sería el proyecto de Vico de una ciencia sobre la naturaleza de las naciones. Una ciencia que contiene una filosofía y una historia de las costumbres humanas, que es afirmada sobre la base de «la convicción» de que al dar una «serie cambiante de creencias» ha dado «la secuencia de causas, ya sean naturalistas o, más tarde, más racionales, sobre las cuales descansa el desarrollo social, cultural y político». En la lectura de Pompa describe Vico el «aspecto filosófico» de su ciencia como «una serie de razones vinculadas» y el «aspecto histórico» como «una secuencia continua de hechos de humanidad» que se ajustan conforme a estas razones. Y así se señala la concepción nuclear de La Primera Ciencia Nueva: «la historia ideal eterna» sobre la que corren en el tiempo todas las historias particulares de las naciones. ${ }^{41}$ De tal modo, es característica principal de la filosofía política de Vico la teoría de un patrón que se muestra repetitivo en la historia de cada nación. Tesis que en la Ciencia nueva primera, a diferencia de las dos siguientes, desemboca en la actitud optimista de la Conclusión de la obra, que envuelve la idea de un acmé de las naciones. Lo cual no significa que se tenga una visión de la ciencia de Vico como «una ciencia predictiva», pues esa

37. L. Pompa, Introduction, cit., p. xix. La tesis de que el conocimiento histórico presupone una teoría acerca de la naturaleza humana está también presente en el libro de L. PoMPA, Human nature and historical knowledge. Hume, Hegel and Vico, Cambridge U.P., Cambridge-Nueva York, 1990 (2002 reed.). La confrontación entre determinismo y libertad o, de otro modo, entre necesidad y contingencia en la ciencia de Vico, fue objeto de la contribución de Pompa al Congreso Internacional sobre Vico celebrado en Sevilla en octubre de 1999: L. PomPA, «Necessity and Contingency in Vico's Philosophy and History of Humanity», en E. Hidalgo-Serna, M. Marassi, J.M. SEvilla, J. Villalobos (Eds.), Pensar para el nuevo siglo. Giambattista Vico y la cultura europea, Ed. La Città del Sole, Nápoles, 2001, 3 vols., II, pp. 513-536.

38. L. POMPA, Introduction, cit., ibídem.

39. Cfr. ibíd., p. xx.

40. Ibíd., p. xxv.

41. Cfr. ibíd., pp. xxviii-xxix. Vid. § 520 en Opere de G. Vico, a cargo de A. BATTistini (A. Mondadori Ed., Milán, 1990, 2 vols.), II, p. 1.220; y en p. 288 de la citada edición de Pompa. 
concepción dependería de un determinismo histórico; mientras que para Vico la operación causal en la "historia ideal eterna", en el mundo real «depende de las circunstancias contingentes en el mundo». Si se dan ciertas circunstancias, la «Ciencia de Vico» podría, al igual que otras ciencias, predecir qué sucederá; «pero no puede predecir que esas circunstancias deban ocurrir, o cuándo lo harán. Solo la historia puede mostrar si las tiene o no». ${ }^{42}$

La edición inglesa de la Ciencia nueva de 1725 constituye todo un homenaje de Pompa al heroísmo científico de Vico, cuya obra, consciente el napolitano de la novedad de la ciencia por él «descubierta», dedicó «Alle accademie dell'Europa le quali in questa età illuminata...». Una dedicatoria no solo con pretensión de reconocimiento de su obra, sino, como dice Battistini, ${ }^{43}$ con intención de revalorizar el sentido comunitario del saber, de vincular -conforme el propio Vico había teorizado- al intelectual con el pueblo del que procede, y encauzar el saber y la ciencia en beneficio de la sociedad y del desarrollo de la humanidad civil. Por tanto, y según Pompa ha interpretado: tratando de vincular el ejercicio teorético viquiano y su modelo epistémico con la realidad social del hombre; como el Prometeo que trae la luz de la ciencia al oscuro mundo humano. Nuestro héroe civil.

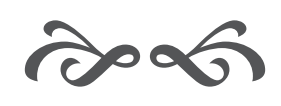

42. Ibíd., p. xxxviii. Cfr. pp. xxxvi-xxxvii.

43. Cfr. "Note" de Battistini en vol. II cit., p. 1.759. 